\title{
Calculations on Countercurrent Electromigration
}

\author{
By G. Breit ${ }^{2}$ and F. L. Friedman
}

\begin{abstract}
The mathematical problems presented by the countercurrent electrolysis method for isotope separation are treated under the simplified assumptions described in the text as (a), (b), (c), (d), (e), and (f) in section II, 2, of this paper. The solutions are worked out in section II, 3, for a tube of finite length. The formulas are put into numerical form in equations $9 \mathrm{e}, 9 \mathrm{f}$, etc. Simpler formulas are obtained in section II, 4, in the approximation of tubes of infinite length. Some of the results are presented in the form of graphs that are described in section III. The orders of magnitude of effects to be expected are discussed in section IV.
\end{abstract}

\section{Introduction}

The countercurrent electromigration method has been found to give isotope separation in the case of potassium $[1,2]^{3}$ and chlorine [3]. In the present report some diffusion problems of this method are treated mathematically. Formulas are given for the calculation of the concentration along the tube. Some typical numerical examples are given to illustrate the use of the formulas and graphs and to show the variation of concentration with time and distance.

\section{Calculations}

\section{Notation}

$n_{l}=$ number of more mobile ions per cubic centimeter (supposedly light).

$n_{h}=$ number of less mobile ions per cubic centimeter (supposedly heavy).

$\mu_{l}, \mu_{h}=$ mobilities of light and heavy ions respectively.

$D_{l}, D_{h}=$ effective diffusion coefficients of light and heavy ions.

$v=$ velocity of liquid flow (positive when toward cathode).

1 This paper will appear in volume 6, division III, of the Manhattan Project Technical Series.

${ }^{2}$ Professor of Physies, University of Wisconsin, now, Yale University. Prof. Breit was on the staff of the National Bureau of Standards in connection with the initiation of the atomic-energy project when this work was done.

${ }^{3}$ Figures in brackets indicate the literature references at the end of this paper.
$E=$ electric field (positive when directed from anode to cathode).

$n_{l}^{\circ}, n_{h}^{\circ}=$ normal values of $n_{l}, n_{h}$.

$\mu=$ common symbol for $\mu_{l}$ or $\mu_{h}$ if distinguishing between them is immaterial.

$D=$ common symbol for $D_{l}$ or $D_{h}$ if distinguishing between them is immaterial.

$x=$ distance along tube measured from anode to cathode.

$V=$ volume of cathode compartment.

$A=$ cross sectional area of tube free to carry electric current.

$\Delta \mu=\mu_{l}-\mu_{h}$.

$L=$ length of tube.

$t=$ time counted from beginning of experiment.

$L^{\prime}=V / A=$ effective length of cathode.

\section{Simplifying Assumptions and Basic Equations}

It is assumed that: (a) The mobilities and the diffusion coefficients have the same values throughout the tube. This is not true, but it is felt that the variation of these quantities may be neglected for the present preliminary estimates. (b) The electric field is assumed to be constant along the tube. This is also not strictly true. As the electric field could be maintained at a constant value, this assumption should not matter much. (c) The cathode is assumed to be stirred perfectly. (d) The concentrations in the anode are supposed to be maintained at their normal 
values. (e) The solution is supposed to be completely dissociated. (f) Forces acting on ions due to space charge effects are neglected.

At a point in the tube having distance, $x$, from the anode the flux of light ions is

$$
\phi_{l}=\left(\mu_{l} E+v\right) n_{l}-D_{l} \frac{\partial n_{l}}{\partial x}
$$

The first term takes account of the combined action of the electric field which gives rise to the migration and of the counter current velocity, $v$. One has, therefore,

$$
\begin{aligned}
& \frac{\partial n_{l}}{\partial t}+\frac{\partial}{\partial x}\left[n_{l}\left(\mu_{l} E+v\right)-D_{l} \frac{\partial n_{l}}{\partial x}\right]=0 \\
& \frac{\partial n_{h}}{\partial t}+\frac{\partial}{\partial x}\left[n_{h}\left(\mu_{h} E+v\right)-D_{h} \frac{\partial n_{h}}{\partial x}\right]=0
\end{aligned}
$$

for the equations expressing equilibrium of the light and heavy ions within the tube. At the cathode compartment $x=L$. Since the liquid inside the cathode is stirred one has

$$
\begin{gathered}
\frac{V}{A} \frac{\partial n_{l}}{\partial t}=\phi_{l}=\left(\mu_{l} E+v\right) n_{l}-D_{l} \frac{\partial n_{l}}{\partial x}(x=L) \\
\frac{V}{A} \frac{\partial n_{h}}{\partial t}=\phi_{h}=\left(\mu_{h} E+v\right) n_{h}-D_{h} \frac{\partial n_{h}}{\partial x}(x=L)
\end{gathered}
$$

It is convenient to introduce

$$
\begin{gathered}
a_{l}=\mu_{l} E+v, a_{h}=\mu_{h} E+v \\
\tau_{l}=\frac{a_{l}^{2}}{D_{l}} t, \tau_{h}=\frac{a_{h}^{2}}{D_{h}} t \\
\xi_{l}=\frac{a_{l}}{D_{l}} x, \xi_{h}=\frac{a_{h}}{D_{h}} x \\
\lambda_{l}=\frac{a_{l}}{D_{l}} L, \lambda_{h}=\frac{a_{h}}{D_{h}} L \\
\gamma_{l}=\frac{V}{A} \frac{a_{i}}{D_{l}}=\frac{V}{A L} \lambda_{l}=\frac{L^{\prime}}{L} \lambda_{l} ; \gamma_{h}=\frac{L^{\prime}}{L} \lambda_{h}
\end{gathered}
$$

This amounts to introducing units that are somewhat more natural for the discussion than the original units. In terms of the natural units one has instead of eq 2 and $2 \mathrm{a}$;

$$
\frac{\partial n_{l}}{\partial \tau_{l}}=\frac{\partial^{2} n_{l}}{\partial \xi_{l}^{2}}-\frac{\partial n_{l}}{\partial \xi_{l}}, \frac{\partial n_{h}}{\partial \tau_{h}}=\frac{\partial^{2} n_{h}}{\partial \xi_{h}^{2}}-\frac{\partial n_{h}}{\partial \xi_{h}}
$$

The conditions for the cathode compartment become

$$
\left(\gamma_{l} \frac{\partial n_{l}}{\partial \tau_{l}}\right)_{\lambda_{l}}=\left(n_{l}-\frac{\partial n_{l}}{\partial \xi_{l}}\right)_{\lambda_{l}},\left(\gamma_{h} \frac{\partial n_{h}}{\partial \tau_{h}}\right)_{\lambda_{h}}=\left(n_{h}-\frac{\partial n_{h}}{\partial \xi_{h}}\right)_{\lambda_{h}}
$$

The problem is defined by equations 3 , 3a, and the conditions

$$
\begin{aligned}
& n_{l}(o, t)=n_{l}^{\circ}, n_{h}(o, t)=n_{h}^{\circ} \\
& n_{l}(x, o)=n_{l}^{\circ}, n_{h}(x, o)=n_{h}^{\circ}
\end{aligned}
$$

which express the fact that at all times the concentrations are normal at the anode and also that at $t=0$ the concentrations are normal throughout the tube.

Since the equations are of the same form for $n_{i}$ and $n_{h}$, the indices $l$ and $h$ may be dropped in most of the work.

\section{Tube of Finite Length}

In this section an exact solution for a tube of finite length is given. The motion of one isotope only is considered and the formulas are supposed to be applied by working out separately the concentrations of the two isotopes. The indices $l, h$ are omitted in the discussion of the behaviour of a single isotope. The equation

$$
\frac{\partial n}{\partial \tau}=-\frac{\partial n}{\partial \xi}+\frac{\partial^{2} n}{\partial \xi^{2}}
$$

is being solved subject to boundary conditions (eq $3 \mathrm{a}, 3 \mathrm{~b}$, and $3 \mathrm{c})$.

For $\tau=\infty$ one has in addition

$$
n(\xi, \infty)=n^{\circ} e^{\xi} .
$$

The solution can be constructed by expanding the initial state in terms of a complete set of functions. For $\gamma=0$ the functions are mutually orthogonal and the method is then well known. For $\gamma \neq 0$ the functions are not mutually orthogonal. It is nevertheless possible to give an explicit usable solution in this case also.

Equation 4 is first transiormed by

$$
n=e^{\xi / 2} N
$$

It assumes the form

$$
\frac{\partial^{2} N}{\partial \xi^{2}}-\frac{N}{4}=\frac{\partial N}{\partial \tau}
$$


The function $n$ is expanded as

$$
n(\xi, \tau)=n^{\circ} e^{\xi}+e^{\xi / 2} \sum a_{i} v_{i}(\xi) e^{-\beta_{i} \tau}
$$

where $v_{\imath}(\xi) e^{-\beta_{i} \tau}$ are solutions of eq $4 \mathrm{c}$ so that

$$
\frac{d^{2} v_{i}}{d \xi^{2}}+\left(\beta_{i}-\frac{1}{4}\right) v_{\imath}=0 .
$$

The functions $v_{i}$ are taken so as to satisfy the boundary conditions

$$
v_{i}(0)=0,\left[\frac{d v_{i}}{d \xi}\right]_{\xi=\lambda}=\left(\beta_{i} \gamma+\frac{1}{2}\right) v_{i}(\lambda)
$$

The first of these corresponds to eq $3 \mathrm{~b}$, the second to eq 3a. The condition in eq $3 \mathrm{c}$ is taken care of by making

$$
2 n^{\circ} \sinh \frac{\xi}{2}+\sum a_{i} v_{i}(\xi)=0
$$

All initial conditions are satisfied automatically as a result of eq $4 \mathrm{f}$ and $4 \mathrm{~g}$. It remains to determine the $a_{i}$.

Combining two previous equations one has

$$
v_{j}(\lambda) v_{i}^{\prime}(\lambda)-v_{j}^{\prime}(\lambda) v_{i}(\boldsymbol{\lambda})=\left(\boldsymbol{\beta}_{j}-\boldsymbol{\beta}_{i}\right) \int_{0}^{\lambda} v_{j}(\xi) v_{i}(\xi) d \xi
$$

Eliminating $v_{i}^{\prime}, v_{j}^{\prime}$ by means of eq $4 \mathrm{f}$, one has for $i \neq j$

$$
\int v_{i}(\xi) v_{j}(\xi) d \xi+\gamma v_{i}(\lambda) v_{j}(\lambda)=0
$$

In order to expand the function

$$
-2 n^{\circ} \sinh (\xi / 2)=f(\xi)=\Sigma a_{i} v_{i}(\xi)
$$

the functions $v_{i}(\xi)$ are first normalized to unity:

$$
\int_{0}^{\lambda} v_{i}^{2}(\xi) d \xi=1
$$

By integration one obtains from eq 5a,

$$
a_{i}-\gamma \sum_{i \neq j} v_{i}(\lambda) v_{j}(\lambda) a_{j}=f_{i}, f_{i}=\int_{0}^{\lambda} v_{i}(\xi) f(\xi) d \xi
$$

The abbreviations

$$
\begin{aligned}
& v_{i}=v_{i}(\lambda), \sigma=\Sigma a_{j} v_{j}=f(\lambda)
\end{aligned}
$$

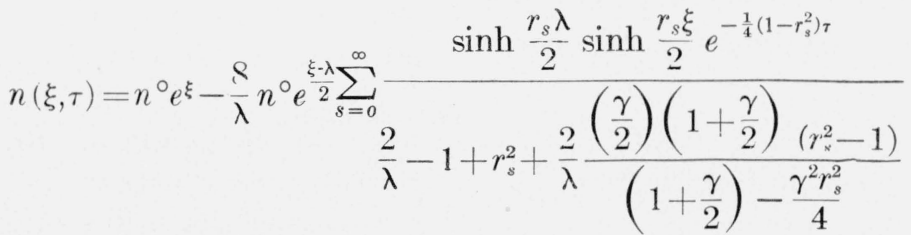

are introduced. In terms of these, eq $5 \mathrm{c}$ gives

$$
a_{i}=\frac{f_{\imath}+\gamma v_{2} \sigma}{1+\gamma v_{i}^{2}}
$$

The boundary conditions in eq $4 \mathrm{f}$ and the normalization in eq $5 b$ are satisfied by

$v_{0}(\xi)=\frac{\sqrt{2 / \lambda \sinh (r \xi / 2)}}{\sqrt{\sinh r \lambda / r \lambda-1}} ; r=\sqrt{1-4 \beta}, r=$ real number

where

determines $r$

Equation $4 \mathrm{c}$ is similar in form to the time dependent wave equation in wave mechanics. Only real $\beta \geqq 0$ need be considered, therefore. Accordingly, only real or pure imaginary values of $r$ are of interest.

For $\gamma \geqq 0, \lambda>0$ eq 6 a has in addition to $r=0$ at most one real root for $r$ and this lies in $0<r<1$. In addition there are pure imaginary roots

$$
r=i \rho_{s}
$$

These satisfy

$$
\tan \left(\frac{\rho_{s} \lambda}{2}\right)=\frac{\rho_{s}}{1+\gamma\left(1+\rho_{s}^{2}\right) / 2}
$$

There is an infinite number of roots $\rho_{s}$ forming a discrete set.

If the velocity of the liquid is higher than the migration velocity then $\lambda$ and $\gamma$ have negative values. From eq 6a one obtains

$$
\lambda=\frac{1}{r} \ln \left[\frac{1+r}{1-r} \frac{1+\gamma(1-r) / 2}{1+\gamma(1+r) / 2}\right]
$$

For $\gamma<-2$, values of $r$ in $0<r<1+2 / \gamma$ give values of $\lambda$ in $-\frac{4 /|\gamma|}{1-2 /|\gamma|}>\lambda>-\infty$. For $\gamma>-2$ there are no real roots for $r$. For $a<0$ there is thus also at most one real root for $r$.

Performing the calculation according to eq $4 \mathrm{~d}$, $5 \mathrm{a}, 5 \mathrm{e}$, and making use of eq $6 \mathrm{a}$ and $6 \mathrm{c}$, it is found 
where the summation covers real and imaginary $r_{s}$. For imaginary $r_{s}$ eq. $6 \mathrm{~b}$ and $6 \mathrm{c}$ are used and $\sinh \left(r_{s} \lambda / 2\right)$ is replaced by $i \sin \left(\rho_{s} \lambda / 2\right)$. As a special case

$$
n(\lambda, \tau)=n^{\circ} e^{\lambda}-\frac{8}{\lambda} n^{\circ} \sum_{s=0}^{\infty} \frac{\frac{r_{\mathrm{s}}^{2}}{1-r_{s}^{2}} e^{-\frac{1}{4}\left(1-r_{s}^{2}\right) \tau}}{\left(\frac{2}{\lambda}-1+r_{s}^{2}\right)\left[\left(1+\frac{\gamma}{2}\right)^{2}-\frac{\gamma^{2}}{4} r_{s}^{2}\right]-\frac{\gamma}{\lambda}\left(1+\frac{\gamma}{2}\right)} \overline{\left(1-r_{s}^{2}\right)}
$$

gives the concentration at the cathode. For large positive $\gamma$ the real root moves toward unity so that $r_{0}=1-\epsilon$ where $\epsilon<<1$. In this limit one has approximately

$$
\gamma=\frac{2 / \epsilon}{e^{\lambda}-1}
$$

The imaginary roots contribute terms in $1 / \gamma^{2}$ and become small for $\gamma \rightarrow \infty$. Equation 7 a gives in this limit

$$
n(\lambda, \tau)=n^{\circ} e^{\lambda}-n^{\circ}\left(e^{\lambda}-1\right) e^{\frac{-\tau}{\gamma\left(e^{\lambda}-1\right)}}
$$

so that the approximate time constant in units $D / a^{2}$ is

$$
\tau^{\circ}=\gamma\left(e^{\lambda}-1\right),
$$

which corresponds to a time constant in seconds

$$
T^{\circ}=\frac{V}{A a}\left(e^{\lambda}-1\right)
$$

If in addition $\lambda<<1$ then

$$
T^{\circ} \cong \frac{V L}{A D}=\frac{L^{\prime} L}{D}
$$

If $a<0$, eq 8 to $8 \mathrm{~d}$ still hold for large values of $-\gamma$.

For a small cathode compartment, one has the simpler formulas:

$$
\begin{aligned}
& n(\xi, \tau)= \\
& n^{\circ} e^{\xi}-\frac{8 n^{\circ}}{\lambda} e^{\frac{\xi-\lambda}{2}} \sum_{s=0}^{\infty} \frac{\sinh \frac{r_{s} \lambda}{2} \sinh \frac{r_{s} \xi}{2} e^{-\frac{1}{4}\left(1-r_{s}^{2}\right) \tau}}{-1+r_{s}^{2}+2 / \lambda} \\
& n(\lambda, \tau)=n^{\circ} e^{\lambda}-\frac{8}{\lambda} n^{\circ} \sum_{s=0}^{\infty} \frac{r_{s}^{2} e^{-\left(1-r_{s}^{2}\right) \tau / 4}}{\left(1-r_{s}^{2}\right)\left(-1+r_{s}^{2}+2 / \lambda\right)}
\end{aligned}
$$

For $\gamma=0, \lambda=2$, the real root is in the process of becoming imaginary, and the corresponding term in the above formulas needs special consideration becoming indeterminate. Making $\lambda=2+\epsilon$ with $\epsilon \rightarrow 0$ it is found that:

$$
\begin{aligned}
& n(\xi, \tau)=n^{\circ} e^{\xi}- \\
& n^{\circ} e^{\frac{\xi-2}{2}}\left\{3 \xi e^{-\beta_{0} \tau}+\sum_{1}^{\infty} \frac{\sin \rho_{s} \sin \left(\rho_{s} \xi / 2\right) e^{-\beta_{s} \tau}}{\left(\rho_{s} / 2\right)^{2}}\right\}
\end{aligned}
$$

$$
n(\lambda, \tau)=n^{\circ} e^{2}-n^{\circ}\left\{6 e^{-\beta_{0} \tau}+\sum_{1}^{\infty} \frac{4}{1+\rho_{s}^{2}} e^{-\beta_{s} \tau}\right\}
$$

In this case the roots are known and are tabulated in Jahncke-Emde [4]. Substitution of numbers gives

$$
\begin{aligned}
& n(\lambda, \tau) / n^{\circ}=e^{2}-6 e^{-\beta_{0} \tau}-0.18876 e^{-\beta_{1} \tau}- \\
& 0.06592 e^{-\beta_{2} \tau}-0.03336 e^{-\beta_{3} \tau}-0.02011 e^{-\beta_{4} \tau}- \\
& 0.01344 e^{-\beta_{5} \tau}-0.00962 e^{-\beta_{6} \tau}-0.00722 e^{-\beta_{7 \tau}}- \\
& 0.00562 e^{-\beta_{8} \tau}-0.004496 e^{-\beta_{9} \tau}-0.00368 e^{-\beta_{10 \tau}}- \\
& 0.00307 e^{-\beta_{11 \tau}}-0.002595 e^{-\beta_{12 \tau} \tau}-0.00223 e^{-\beta_{13} \tau}- \\
& 0.001929 e^{-\beta_{14} \tau}-0.001688 e^{-\beta_{15 \tau} \tau}-0.001489 e^{-\beta_{16} \tau}- \\
& 0.001324 e^{-\beta_{17} \tau}-0.001184 e^{-\beta_{18} \tau}-0.001066 e^{-\beta_{19 \tau}}- \\
& 0.000965 e^{-\beta_{20} \tau}-0.000877 e^{-\beta_{21} \tau}-0.000801 e^{-\beta_{22} \tau}-
\end{aligned}
$$

Also

$$
\begin{aligned}
n(1, \tau)= & n^{\circ} e-\frac{n^{\circ}}{\sqrt{e}}\left\{3 e^{-\beta_{0} \tau}-0.15087 e^{-\beta_{1} \tau}-\right. \\
& 0.04388 e^{-\beta_{2} \tau}+0.02473 e^{-\beta_{3} \tau}+ \\
& 0.01374 e^{-\beta_{4} \tau}-0.00979 e^{-\beta_{5} \tau}- \\
& 0.00664 e^{-\beta_{6} \tau}+0.00522 e^{-\beta_{7} \tau}+ \\
& \left.0.00390 e^{-\beta_{8} \tau}-0.00323 e^{-\beta_{9} \tau}-\ldots .\right\}
\end{aligned}
$$

The $\beta$ 's in eq $9 \mathrm{~d}$ and in $9 \mathrm{e}$ are given by:

$$
\beta_{0}=\frac{1}{4}, \beta_{1}=\frac{1}{0.18876}, \beta_{2}=\frac{1}{0.06592}, \beta_{3}=\frac{1}{0.03336}
$$

In order to see how the cathode volume affects the results, the above solution can be compared with that for $\lambda=2, \gamma=15.2195$. The choice of $\gamma$ in this calculation is made so as to give $r_{0}=0.98$. Approximate values of the $\rho^{\prime}$ s are $\rho_{1}=3.18, \rho_{2}=$ $6.30, \rho_{3}=9.44$. For the cathode, one obtains by means of eq $7 \mathrm{a}$,

$$
\begin{aligned}
\frac{n}{n^{\circ}=} & 7.3891-6.3885 e^{-0.0099 \tau}- \\
& 0.00043 e^{-2.78 \tau}-0.00004 e^{-10.2 \tau}-\ldots .
\end{aligned}
$$

which shows that in this case only the first term having the time constant $T=101 D / a^{2}$ is of real importance. The approximation in eq $8 \mathrm{~b}$ gives $T=97 \mathrm{D} / \mathrm{a}^{2}$ and is suitable for an approximate estimate for this $\gamma$ and $\lambda$. Because of the more rapid convergence of the series, calculations with large $\gamma$ are easier than with small $\gamma$. 
Another example of the application of eq $7 \mathrm{a}$ is $\lambda=2, r_{0}=0.90, \quad \gamma=2.6995$ for which $\rho_{1}=3.331$, $\rho_{2}=6.394$, and

$$
\begin{aligned}
\left(\frac{n}{n^{\circ}}\right)_{\xi=\lambda}= & 7.3891-6.376 e^{-0.0475 \tau}-0.01132 e^{-3.025 \tau}- \\
& 0.00115 e^{-10.47 \tau}-0.00025 e^{-22.9 \tau}-\ldots .
\end{aligned}
$$

An example for negative a may also be given: $\lambda=-1, r_{0}=0.50, \gamma=-8.111, \rho_{1}=6.359, \rho_{2}=12.605$, which gives

$$
\begin{aligned}
\left(\frac{n}{n^{\circ}}\right)_{\xi=\lambda}= & 0.36788+0.6319 e^{-\frac{3 \tau}{16}}+ \\
& 0.00028 e^{-10.36 \tau}+\ldots .
\end{aligned}
$$

A consideration of these examples shows that for a rather large range of values of $\gamma$, the term in $e^{-\frac{1-r_{0}^{2}}{4} \tau}$ is the last important one. The relation $\tau^{\circ}=\gamma\left(e^{\lambda}-1\right)$ for its time constant can be improved on by using a graphical or numerical solution of

and setting

$$
\tanh \frac{r \lambda}{2}=\frac{r}{1+\gamma\left(1-r^{2}\right) / 2}
$$

$$
\tau^{\circ}=\frac{4}{1-r_{0}^{2}}
$$

\section{Tubes of Infinite Length}

Substitution of different $\tau$ into eq 9d, 9e shows that the concentration at the cathode builds up much more rapidly than in the middle of the tube. For times appreciably smaller than the time constant the effect of the anode is, therefore. not important, and the calculation of exact solutions involves a needless amount of work. The initial stages of the building up of the concentration can be studied by supposing that the tube is infinitely long because the solution for an infinite tube gives practically zero concentration at the anode.

In discussing a tube of infinite length it is convenient to measure lengths from the cathode introducing

$$
\eta=\lambda-\xi
$$

The differential equation is

$$
\frac{\partial^{2} n}{\partial \eta^{2}}+\frac{\partial n}{\partial \eta}=\frac{\partial n}{\partial \tau}
$$

and the boundary condition at the cathode is convenientlý expressed in terms of

for which

$$
m=\frac{\partial n}{\partial \eta}+n
$$

$$
\left(\gamma \frac{\partial n}{\partial \tau}-m\right)_{\eta=0}=\left(\gamma \frac{\partial m}{\partial \eta}-m\right)_{\eta=0}=0
$$

The quantity $m$ is the flux density of ions divided by $a$. It has proved convenient to use $m$ in constructing the solution because $m-\gamma \frac{\partial m}{\partial \eta}$ has the simple initial condition of being 1 throughout the length of the tube. It is thus possible to construct first the solution for $\gamma=0$, to equate the $m$ ob tained for $\gamma=0$ to $m-\gamma \frac{\partial m}{\partial \eta}$ and derive $m$ for any $\gamma$. From $m$, one obtains $n$ by quadratures.

For $a>0$, one finds

$$
\begin{aligned}
\frac{n-n^{\circ}}{n^{\circ}=} & \frac{\sqrt{\tau / \pi}}{1+\gamma} e^{-\frac{(\eta+\tau)^{2}}{4 \tau}}-\frac{1}{2}\left[1-\Phi\left(\frac{\eta+\tau}{2 \sqrt{\tau}}\right)\right]+ \\
& \frac{e^{-\eta}}{2} \frac{1-\eta+\frac{1}{1+\gamma}}{1+\gamma}\left[1-\Phi\left(\frac{\eta-\tau}{2 \sqrt{\tau}}\right)\right]+ \\
& \frac{(2+\gamma) \gamma}{2(1+\gamma)^{2}} e^{\frac{\eta+\tau}{\gamma}+\frac{\tau}{\gamma^{2}}}\left[1-\Phi\left(\frac{\eta+\tau}{2 \sqrt{\tau}}+\frac{\sqrt{\tau}}{\gamma}\right)\right]
\end{aligned}
$$

where

$$
\Phi(x)=\frac{2}{\sqrt{\pi}} \int_{0}^{x} e^{-x^{2}} d x, \Phi(-x)=-\Phi(x) .
$$

For $\eta=0 \mathrm{i}$. e. for the cathode the above formula is somewhat shorter:

$$
\begin{aligned}
\left(\frac{n-n^{\circ}}{n^{\circ}}\right)_{\eta=0}= & \frac{\tau+1+\gamma+\frac{1}{1+\gamma}}{2(1+\gamma)} \Phi\left(\frac{\sqrt{\tau}}{2}\right)+ \\
& \frac{(2+\gamma) \gamma}{2(1+\gamma)^{2}} e^{\frac{\tau}{\gamma}+\frac{\tau}{\gamma^{2}}}\left[1+\Phi\left(\frac{\sqrt{\tau}}{2}+\frac{\sqrt{\tau}}{\gamma}\right)\right]+ \\
& \frac{\sqrt{\tau / \pi}}{1+\gamma} e^{-\tau / 4}+\frac{\tau}{2(1+\gamma)}-\frac{\gamma(\gamma+2)}{2(1+\gamma)^{2}}
\end{aligned}
$$

and for $\gamma=0$, the following simple form obtains:

$$
\begin{gathered}
\frac{n-n^{\circ}}{n^{\circ}}=\sqrt{\tau / \pi} e^{-\frac{(\eta+\tau)^{2}}{4 \tau}}-\frac{1}{2}\left[1-\Phi\left(\frac{\eta+\tau}{2 \sqrt{\tau}}\right)\right]+ \\
\frac{e^{-\eta}}{2}(\tau-\eta+1)\left[1-\Phi\left(\frac{\eta-\tau}{2 \sqrt{\tau}}\right)\right]
\end{gathered}
$$


The values of $\Phi(x)$ are available in Jahnke-Emde [4] in good detail and numerical calculation by means of eq. 11, 11b, and $11 \mathrm{c}$ is straightforward. A convenient form of $m$ is

$$
\begin{aligned}
\frac{m}{n^{0}}= & \frac{1}{2}+\frac{1}{\sqrt{\pi}} \int_{0}^{(\eta+\tau) / 2 \sqrt{\tau}} e^{-x^{2}} d x- \\
& \frac{e^{-\eta}}{\sqrt{\pi}(1+\gamma)} \int_{(\eta-\tau) / 2 \sqrt{ } \frac{-}{\tau}}^{\infty} e^{-x^{2}} d x+ \\
& \frac{2+\gamma}{\sqrt{\pi}(1+\gamma)} e^{(\eta+\tau) / \gamma+\tau / \gamma^{2}} \int_{(\eta+\tau) / 2 \sqrt{\tau}+\frac{\sqrt{\tau}}{\gamma}}^{\infty} e^{-x^{2}} d x .
\end{aligned}
$$

For $a<0, \lambda, \eta, \gamma$ are negative and $\tau$ is positive. In place of eq 11, 11b, 11c, and 11d one has

$$
\begin{array}{r}
\frac{n-n^{\circ}}{n^{\circ}}=-\frac{\sqrt{\tau / \pi}}{1+\gamma} e^{-(\eta+\tau)^{2 / 4 \gamma}}-\frac{1}{2}\left[1+\Phi\left(\frac{\eta+\tau}{2 \sqrt{\tau}}\right)\right]+ \\
\frac{e^{-\eta}}{2}\left[\frac{\tau-\eta}{1+\gamma}+\frac{1}{1+\gamma^{2}}\right]\left[1+\Phi\left(\frac{\eta--\tau}{2 \sqrt{\tau}}\right)\right]+ \\
\frac{(2+\gamma) \gamma}{2(1+\gamma)^{2}} e^{\frac{\tau+\eta}{\gamma}+\frac{\tau}{\gamma^{2}}}\left[1+\Phi\left(\frac{\eta+\tau}{2 \sqrt{\tau}}+\frac{\sqrt{\tau}}{\gamma}\right)\right] \\
\left(\frac{n-n^{\circ}}{n^{\circ}}\right)_{\eta=0}=-\frac{\sqrt{\tau / \pi}}{1+\gamma} e^{-\tau / 4}-\frac{1}{2}\left[1+\Phi\left(\frac{\sqrt{\tau}}{2}\right)\right]+ \\
\frac{1}{2}\left[\frac{\tau}{1+\gamma}+\frac{1}{(1+\gamma)^{2}}\right]\left[1-\Phi\left(\frac{\sqrt{\tau}}{2}\right)\right]+ \\
\quad \frac{(2+\gamma) \gamma}{2(1+\gamma)^{2}} e^{\frac{\tau}{\gamma}+\frac{\gamma}{\gamma^{2}}}\left[1+\Phi\left(\frac{\sqrt{\tau}}{2}+\frac{\sqrt{\tau}}{\gamma}\right)\right]
\end{array}
$$

and for $\gamma=0$

$$
\begin{aligned}
\left(\frac{n-n^{\circ}}{n^{\circ}}\right)_{\gamma=0}= & -\frac{\sqrt{\tau}}{\sqrt{\pi}} e^{-\frac{(\eta+\tau)^{2}}{2 \sqrt{\tau}}}-\frac{1}{2}\left[1+\Phi\left(\frac{\eta+\tau}{2 \sqrt{\tau}}\right)\right]+ \\
& \frac{e^{-\eta}}{2}(\tau-\eta+1)\left[1+\Phi\left(\frac{\eta-\tau}{2 \sqrt{\tau}}\right)\right]
\end{aligned}
$$

while for arbitrary $\gamma<0$

$$
\begin{aligned}
& \frac{m}{n^{0}}=\frac{1}{2}-\frac{1}{\sqrt{\pi}} \int_{0}^{(\eta+\tau) / 2 \sqrt{\tau}} e^{-x^{2}} d x- \\
& \frac{e^{-\eta}}{\sqrt{\pi}(1+\gamma)} \int_{-\infty}^{(\eta-\tau) / 2 \sqrt{\tau}} e^{-x^{2}} d x+ \\
& \frac{2+\gamma}{\sqrt{\pi}(1+\gamma)} e^{\frac{\eta+\tau}{\gamma}+\frac{\tau}{\gamma^{2}}} \int_{-\infty}^{(\eta+\tau) / 2 \sqrt{\tau}+\sqrt{\tau} / \gamma} e^{-x^{2}} d x
\end{aligned}
$$

The verification of the above equations by substitution into the differential equation and the boundary conditions is straightforward and is not reproduced in this report.

\section{Description of Figures}

In figure 1 there is shown the dependence of $(n-n)^{\circ} / n^{\circ}$ on $\tau$ at the cathode for a tube of infinite length. The curve for zero cathode volume $(\gamma=0)$ is parabolic in shape at the origin. The initial rate at which the concentration builds itself up is seen to be very high. This is because the cathode has zero volume so that the initial rate is infinite. As time goes on the material concentrated at the end of the tube diffuses through a volume proportional to $\sqrt{\tau}$. The rate at which the concentrated material is being fed to this volume

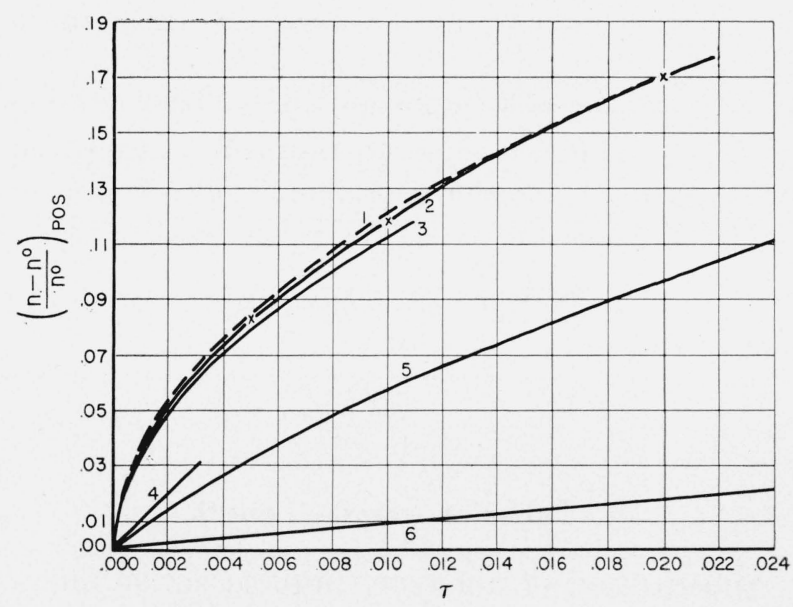

Figure 1.

$\lambda=\infty, n=0 ; x$ are points from $\lambda=2, \gamma=0, \dot{\xi}=2$.

1. Parabola $0.17 \sqrt{\tau / 0.0200} ; 2 . \gamma=0 ; 3$. osculating parabola $2 \sqrt{\tau / \pi} ; 4$. tangent; $5 \gamma=0.1 ; 6 . \gamma=1$

is proportional to $\tau$ and the concentration is therefore proportional to $\tau / \sqrt{\tau}=\sqrt{\tau}$. This is, of course, not an exact consideration, because the rate at which the concentrated material is being fed to the end of the tube depends on the concentration at neighboring places. The parabolic approximation is not bad, however, as is seen in figure 1 , where the dotted curve above the curve for $\gamma=0$ is a parabola $0.17 \sqrt{\tau / .0200}$, chosen so as to give a good overall fit and the dotted curve below the curve for $\gamma=0$ is the osculating parabola $2 \sqrt{\tau / \pi}$. The latter curve makes a second-degree contact with the curve for $\gamma=0$. In figure 1 there are shown also, as crosses, points that correspond 
to $\lambda=2, \xi=2, \gamma=0$. These are seen to fall on the curve $\lambda=\infty, \xi=2, \gamma=0$ to within the accuracy of the drawing. A close agreement of the two curves can be expected because the diffusion distance in natural units $(D / a)$ is of the order $\sqrt{\tau} \sim 0.15$ for the larger $\tau$ in figure 1, while the length of the tube in the same units is 2 . The time in figure 1 may thus be considered so short that the concentrated solution has not yet diffused to the anode. It is satisfactory in this case to make computations of the initial stage of the enrichment on the simplified assumption of an infinitely long tube $(\lambda=\infty)$.

Figure 2 corresponds to negative $a$ i. e. to the removal of ions from the cathode end. In this case $n-n^{\circ}$ is negative and, therefore, $-\left(n-n^{\circ}\right) / n^{\circ}$ is plotted as ordinate. A large ordinate corresponds to a small concentration in this figure and the cathode volume is zero. For $\tau=1$, the concentration is reduced to about $1 / 3$ of its original value. The lower curve is drawn on an expanded time scale so as to show more detail in the initial value.

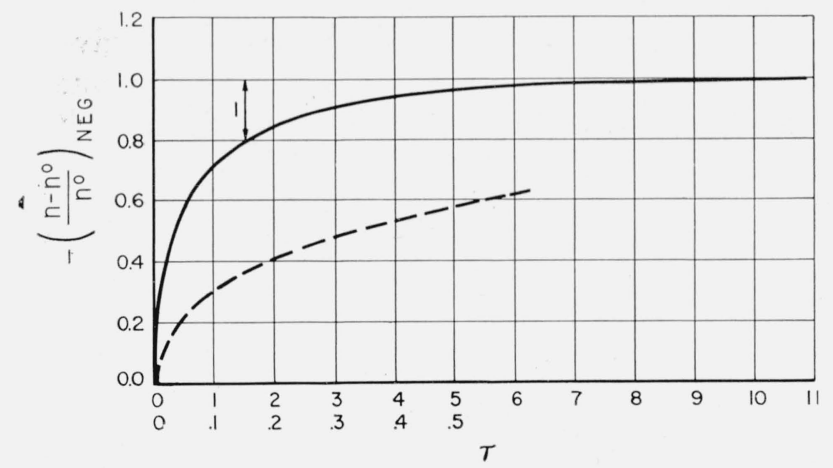

Figure 2.

$\lambda=\infty, \gamma=0, n=0$. 1. Remaining concentration divided by $n^{\circ}=n / n^{\circ}$

In figure 3 a comparison is made between the relative enrichment obtained by having the countercurrent balance exactly one or the other ion type that it is desired to separate. In this and some succeeding figures a positive $a$ that corresponds to feeding an isotope into the cathode is referred to by the subscript "pos" and a negative $a$ that corresponds to washing an isotope out of the cathode is similarly referred to by the subscript "neg". The lower curve in figure 3 corresponds to having the countercurrent of proper strength to balance the migration velocity of the slower isotope. If the initial concentrations of the more

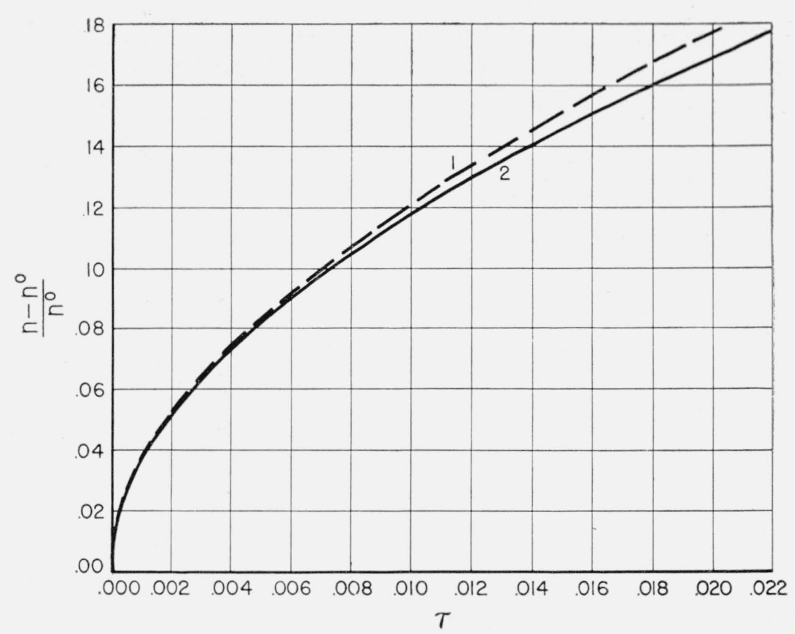

Figure 3.

$$
\lambda=\infty, \gamma=0, n=0, \quad 1 . \frac{1}{1+\left(\frac{n-n^{\circ}}{n^{\circ}}\right)_{\text {ueg }}}-1: 2 .\left(\frac{n-n^{\circ}}{n^{\circ}}\right)_{\text {po: }} .
$$

mobile and less mobile isotopes are $n_{l}^{\circ}, n_{h}^{\circ}$ respectively, then at the time $t$ this ratio is

$$
\frac{n_{l}}{n_{h}}=\frac{n_{l}^{\circ}}{n_{h}^{\circ}}\left[1+\left(\frac{n-n^{\circ}}{n^{\circ}}\right)_{p o s}\right]
$$

and the factor by which this concentration is increased is unity, plus the quantity plotted for the lower curve.

If, on the other hand, the countercurrent is adjusted to balance the more mobile isotope, then the isotope of smaller migration velocity is being drawn out from the cathode and after a time $t$

$$
\begin{aligned}
\frac{n_{l}}{n_{h}}= & \frac{n_{l}^{\circ}}{n_{h}^{\circ}\left[1+\left(\frac{n-n^{\circ}}{n^{\circ}}\right)_{n e q}\right]}= \\
& \frac{n_{l}^{\circ}}{n_{h}^{\circ}}\left\{1+\left[-1+\frac{1}{1+\left(\frac{n-n^{\circ}}{n^{\circ}}\right)_{n e g}}\right]\right\}
\end{aligned}
$$

The quantity in square brackets is plotted as the upper curve in figure 3. It represents, as before, the ratio of concentrations divided by its initial value and minus 1 :

$$
\frac{n_{l} / n_{h}}{n_{?}^{\circ} / n_{h}^{\circ}}-1
$$

It will be noted that for quite a while there is no marked difference in the two ways of adjusting the counter flow that have been considered.

In figure 4 five curves are shown. All of them are for a tube of infinite length. The lower pair 
of curves is for $|\gamma|=0.1$. In this pair the upper curve corresponds to an adjustment of counter flow corresponding to eq $13 \mathrm{a}$, the lower to an adjustment corresponding to eq 13 . The less mobile isotope is being pumped out of the cathode for the upper curve, the more mobile isotope is being pumped into the cathode for the lower.

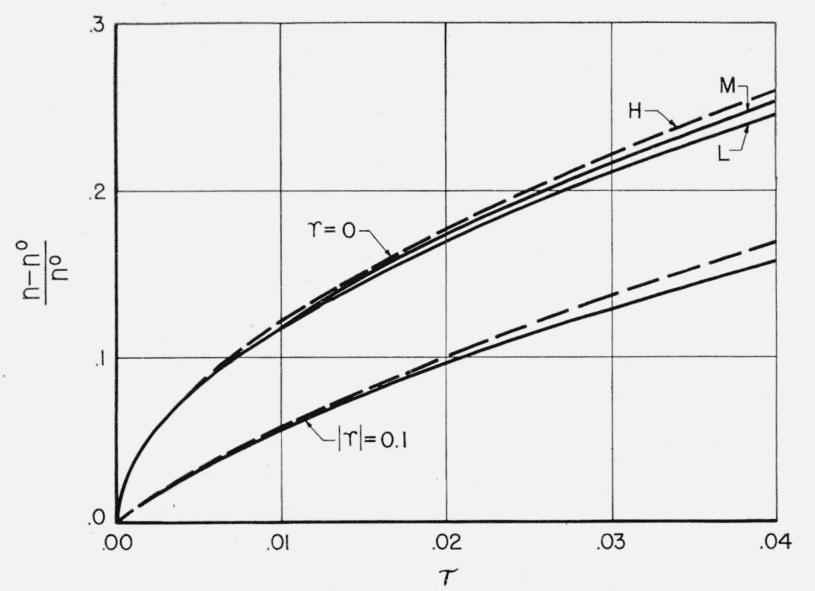

Figure 4.

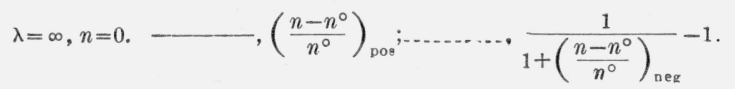

The three upper curves in figure 4 are for a tube of infinite length and zero cathode volume. Among these the highest curve, $H$, corresponds to countercurrent adjustment in eq $13 \mathrm{a}$, the lowest to eq 13 . The curve in between, curve $M$, corresponds to a countercurrent adjustment in which the arithmetic mean of the migration velocities of the more mobile and less mobile isotopes is equal to the velocity of the liquid.

The value of $a$ for eq $13 \mathrm{a}$ is $-\left|\mu_{l}-\mu_{h}\right| E$ and for eq 13 it is $+\left|\mu_{l}-\mu_{h}\right| E$. In the computation for the middle curve in the upper set of three in figure 4 the value of $a$ is $\pm\left|\mu_{l}-\mu_{h}\right| E / 2$ and in accordance with eq $2 \mathrm{e}$, the value of $\tau$ for a given $t$ is $1 / 4$ of what it is for $a= \pm\left|\mu_{l}-\mu_{h}\right| E$. For this reason the abscissae for curve $M$ were made to be $4 \tau$. In this way the same value of the abscissae for the curves $H, M$, $L$ corresponds to the same time, $t$.

The curve, $M$, was computed by plotting $\left[\left(n / n^{\circ}\right)_{\mathrm{pos}} /\left(n / n^{\circ}\right)_{\mathrm{neg}}\right]-1$ as ordinate and $4 \tau$ as abscissae. It is seen that the curve $M$ falls nearly half way between $H$ and $L$. This indicates that the exact adjustment of the countercurrent does not critically affect the ratio of the abundances of the two isotopes as long as there is an approximate balance of migration velocity and counter flow. With the idealized conditions assumed here, the relative enrichment in the cathode is changed more rapidly by having the counter flow too high rather than too low.

The way in which the three typical adjustments of countercurrent give about the same result for small enrichments can be illustrated also in terms of a formula for small enrichments for $\gamma=0$. One has approximately

$$
\left(n / n^{\circ}\right)_{\mathrm{pos}} \cong 1+2 \sqrt{\tau / \pi},\left(n / n^{\circ}\right)_{\mathrm{neg}} \cong 1-2 \sqrt{\tau / \pi}
$$

so that

$$
\left(\frac{n-n^{\circ}}{n^{\circ}}\right)_{\mathrm{pos}} \cong \frac{1}{1+\left(\frac{n-n^{\circ}}{n^{\circ}}\right)_{\mathrm{neg}}}-1 \cong 2 \sqrt{\tau / \pi} \quad(H, L)
$$

which shows the approximate equivalence of countercurrent adjustments, $H$ and $L$. Also, for the countercurrent adjustments of the curve $M$

$$
\frac{1+2 \sqrt{\tau / \pi}}{1-2 \sqrt{\tau / \pi}}-1 \cong \frac{\left(n / n^{\circ}\right)_{\mathrm{nos}}}{\left(n / n^{\circ}\right)_{\mathrm{neg}}}-1 \cong 2 \sqrt{4 \tau / \pi}
$$

so that the quantity $M$ is the same function of $4 \tau$ as $H, L$ are of $\tau$.

For $V \not \equiv 0$ a change in the countercurrent gives a change in $\gamma$. For small $t$ the three types of countercurrent adjustment give nearly the same result. In this case

$$
\left(\frac{n}{n^{\circ}}\right)_{\mathrm{pos}} \cong 1+\frac{\tau}{\left|\gamma_{0}\right|}
$$

where $\gamma_{0}=V\left(\mu_{l}-\mu_{h}\right) E / A D$. Similarly

$$
\left(\frac{n}{n^{\circ}}\right)_{\mathrm{neg}} \cong 1-\frac{\tau}{\left|\gamma_{0}\right|} .
$$

For the arithmetic mean countercurrent adjustment,

$$
\frac{\left(n / n^{\circ}\right)_{\text {pos }}}{\left(n / n^{\circ}\right)_{\text {neg }}}-1 \cong \frac{1+\frac{2 \tau}{\left|\gamma_{0}\right|}}{1-\frac{2 \tau}{\left|\gamma_{0}\right|}}-1=\frac{4 \tau}{\left|\gamma_{0}\right|}
$$

and the result is nearly the same as if the countercurrent were adjusted to keep one of the isotopes stationary with respect to the tube.

In figure 5 the behaviour of a tube of infinite length is compared with that of a tube of finite length in the special case of zero cathode volume. The concentration of an isotope at the cathode end is plotted against $\tau$ in both cases. The equilibrium concentration for the tube of finite length is taken to be $e^{2}=7.4$. Up to $\tau=2$, there is 


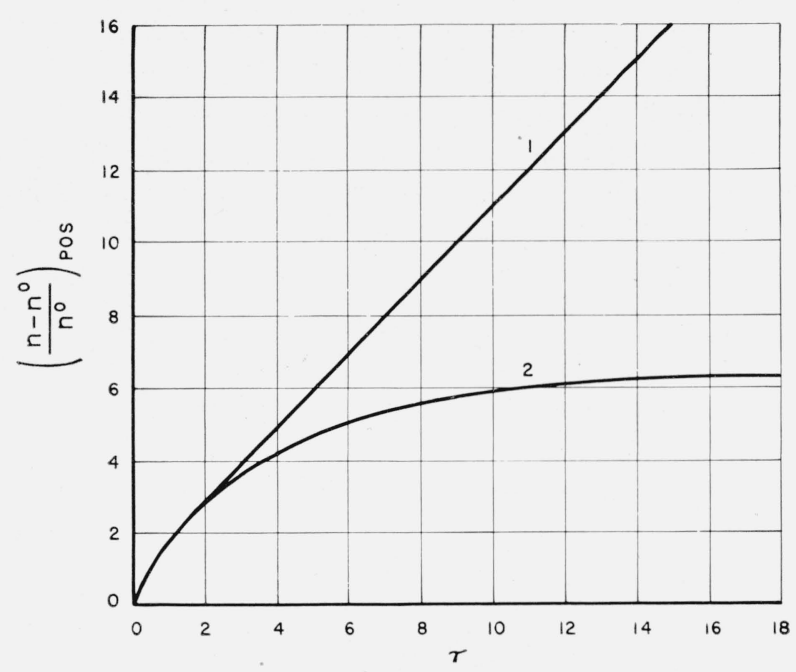

Figure 5.

$\gamma=0, n=0 . \quad 1 . \lambda=\infty, \quad 2 . \lambda=2$.

not much difference between the two tubes. The concentration increases to about four times the initial value at this $\tau$. For $\tau>4$, the slope of the curve for the tube of finite length decreases rapidly so that it will not pay to run above this value. The comparison of $\lambda=2$ with an infinitely long tube has already been made for small $\tau$ in figure 1 . The initial stage of the divergence between the two curves can be seen in figure 6 .

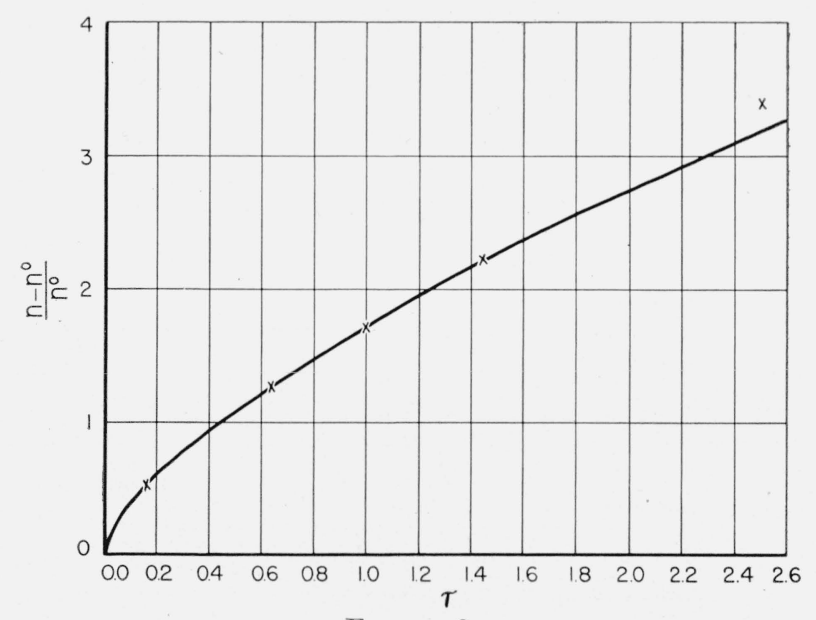

Figure 6.

$\gamma=2, \lambda=2, \xi=2 . x$ are for $\lambda=\infty$.

In figure 7 the dependence of concentration of a single isotope on $\tau$ is illustrated for $\gamma=0$ at different points along a tube of infinite length. The migration velocity is supposed to be greater than the countercurrent corresponding to a positive $a$. At the cathode end the concentration increases steeply, being proportional to $\sqrt{ } \bar{\tau}$ for small $\tau$. For

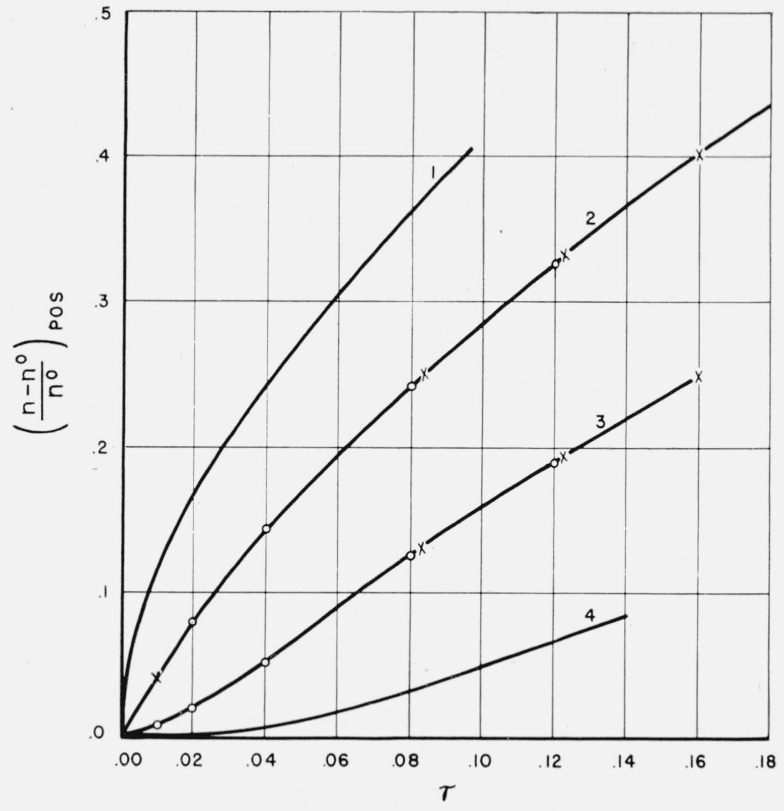

FIgURE 7.

$\gamma=0 . x$ are from $\lambda=\infty ; 0$ are from $\lambda=2.1 . n=0.00 ; 2 . n=0.10,3 . n=0.25$; 4. $n=050$.

points farther away from the cathode the initial portion of the curve is flat and is followed by an approximately linear rise. The initial flat stage can be thought of as corresponding to the time during which the concentration that first develops at the cathode diffuses to the point under consideration. In figure 7 there are also shown points that correspond to a tube of finite length with $\lambda=2$. These are seen to fall very closely on the curve for $\lambda=\infty$. This is to be expected, because for the small $\tau$ covered in this figure there is practically no effect at the anode end so that it does not matter whether the concentration at the anode is maintained at its initial value or not.

In figures 8 and 9 there is illustrated the varia-

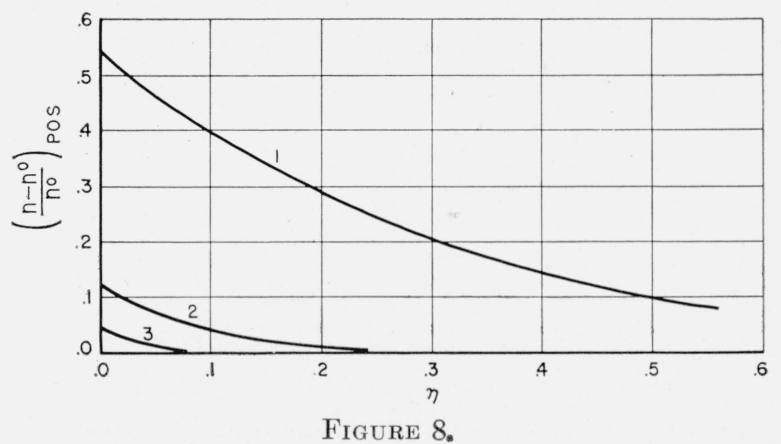

$\gamma=0 . \quad 1 . \tau=0.16 ; 2, \tau=0.010 ; 3, \tau=0.0016$. 


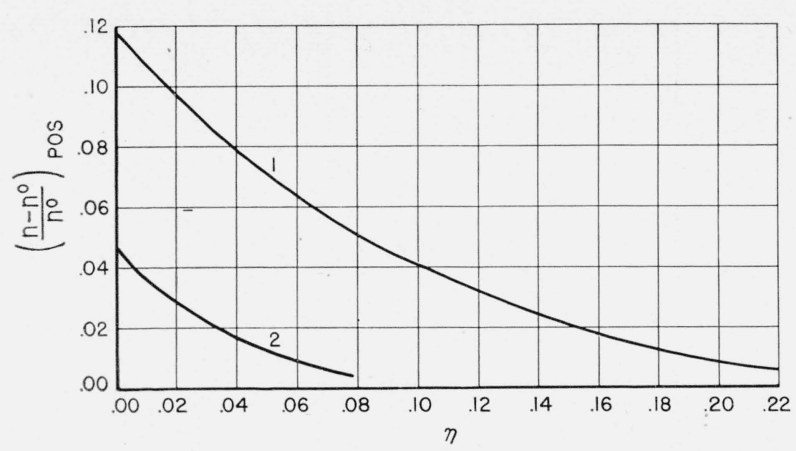

Figure 9.

$\gamma=0 . \quad$ 1. $\tau=0010: 2 . \quad \tau=0.0016$.

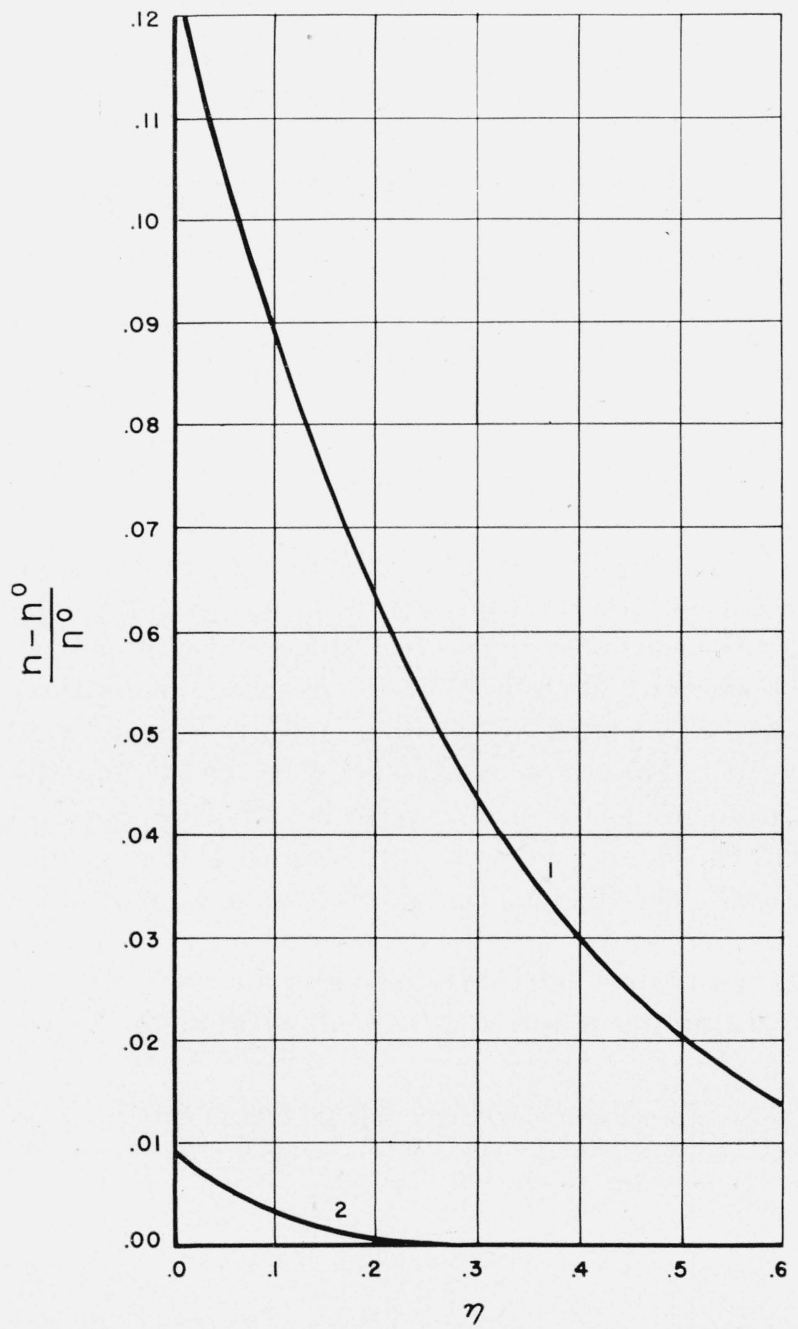

Figure 10.

$\gamma=1 . \lambda=\infty . \quad 1, \quad \tau=0.16 .2 . \quad \tau=0.01$.

tion with distance of $\left[\left(n-n^{\circ}\right) / n^{\circ}\right]$ pos i. e. of the fractional increase of concentration for the case of feeding the isotope towards the cathode. The cathode volume was taken to be zero for these figures, and the tube is infinitely long.

In figure 10 the variation of concentration with distance is illustrated for a finite cathode volume corresponding to $\gamma=1$. The tube is assumed infinitely long and $a>0$ for this figure.

In figure 11 there is illustrated the variation of concentration along the tube for the constants used in eq 9f. The computation can be made by means of formula 9. The term $n^{0} e^{\xi}$ outside the sum is referred to as the "equilibrium term". The way in which the successive terms in the series converge is illustrated by the three curves. The first curve is marked "equilibrium $+\left(r_{0}\right)$ ". It represents the effect of the term $n^{\circ} e^{\xi}$ and of the first term in the summation in eq 9 . The second curve is marked "equilibrium $+\left(r_{0}\right)+\left(\rho_{1}\right)$ ". It includes, in addition to the two previous terms, also the second term in the summation which is due to the root $\rho_{1}$. This curve is already a fair approximation to the answer. The third curve includes the effect of the root $\rho_{2}$ and is marked "equilibrium $+\left(r_{0}\right)+\left(\rho_{1}\right)+\left(\rho_{2}\right)$ ". It is practically the answer. The special value, $\tau=0.16$, was used for all curves in this figure.

In figure 12 there is illustrated the superposition of terms for $\lambda=-1, \gamma=-8.1106, \tau=0.16$ as in eq $9 \mathrm{~g}$.

In this case the isotope is being pumped out of the cathode. The convention regarding labeling curves is the same as in figure 10. The convergence

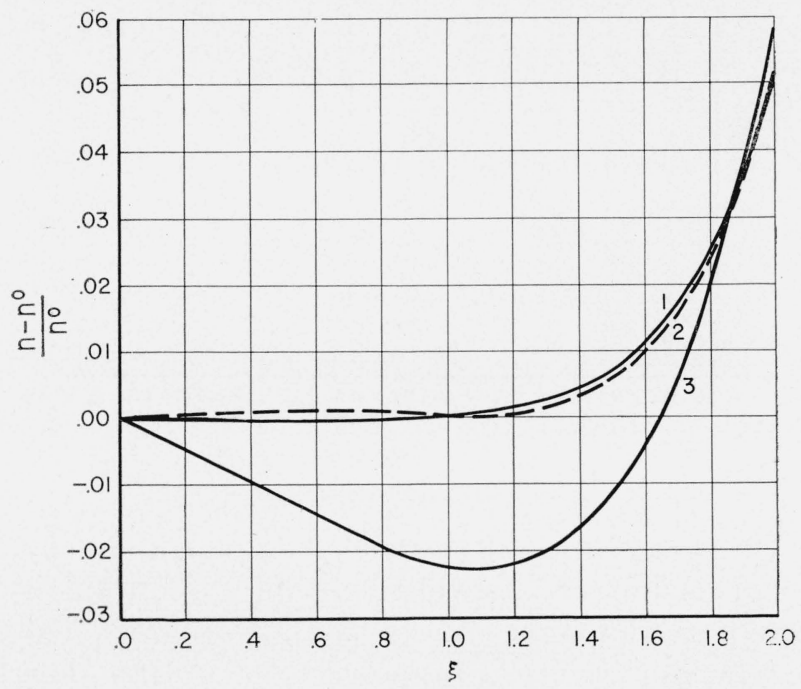

Figure 11.

$\lambda=2, \gamma=2.6996, \tau=0.16 . \quad$ 1. Equilibrium $+\left(r_{0}\right)+\left(\rho_{1}\right)+\left(\rho_{2}\right) ; 2$. equilibrium + $\left(r_{1}\right)+\left(\boldsymbol{b}_{1}\right), 3$. equilibrium $+\left(r_{0}\right)$. 


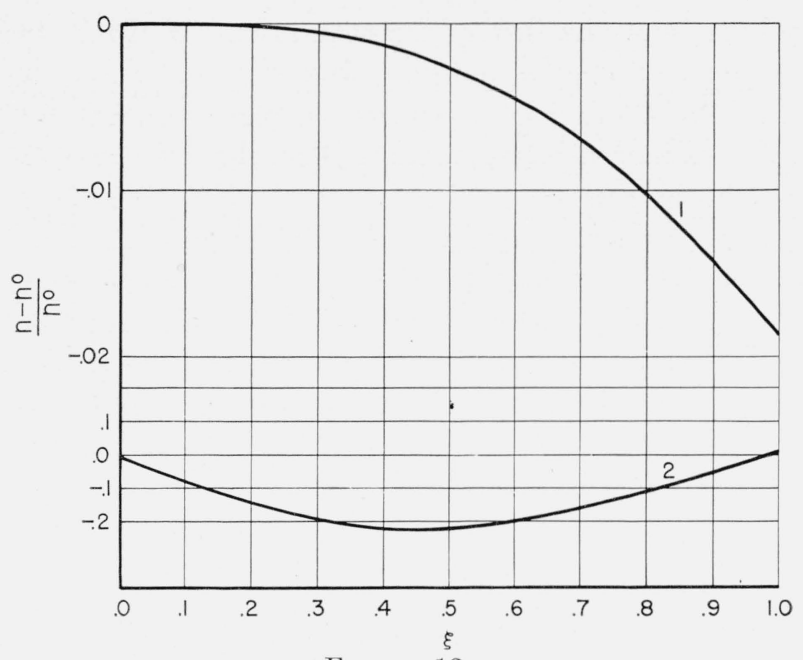

Figure 12.

$\lambda=-1, \gamma=-8.1106, \tau=0.16$. 1. Equilibrium $+\left(r_{0}\right) ; 2 . \quad\left(\rho_{1}\right) \times 10^{4}$ contribution.

is very good. The curve for "equilibrium $+\left(r_{0}\right)$ " is practically the answer. The contribution due to the term in $\rho_{1}$ multiplied by $10^{4}$ is shown also in the lower part of the figure.

In figure 13 small $\lambda$ and large $\gamma$ are employed, $\lambda=1 / 5$ and $\gamma=24.022$. The convergence is so rapid that even at $\tau=0$ only the real root need be considered. For $\tau=1.6$, ten times the $\tau$ used in the two preceding figures, the distribution along the tube is not far from linear.

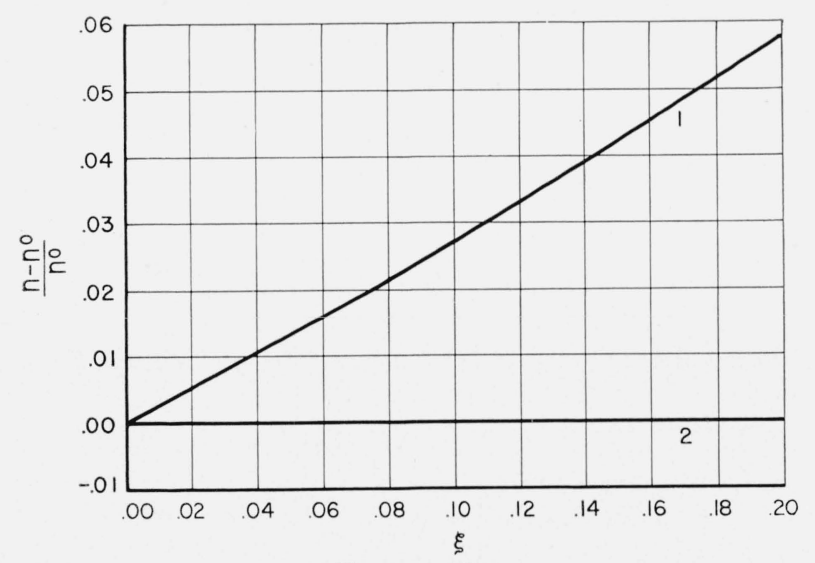

Figure 13.

$\lambda=2, \gamma=24.022$. Equilibrium term $+\left(r_{0}\right)$ term in beth curves. 1. $\tau=1.6$ 2. $\tau=0$.

\section{Discussion}

\section{Orders of Magnitude.}

It appears possible that the difference in mobilities is of the order of $\left(\sqrt{M_{h} / M_{l}}-1\right) \mu$ where $\mu$ is the mobility and $M_{h}, M_{l}$ are the masses of the ions. For $K^{+}$, the experimental value of $\mu$ is $\dot{\sim} 6.6 \times 10^{-\frac{\mathrm{cm}^{2}}{\mathrm{v}-\mathrm{sec}}}$. The effect of convection on $D_{h}, D_{l}$ due to thermal effects is not known. A lower limit for $D_{l}, D_{h}$ can be obtained from the relations

$$
\mu_{l}=\frac{e}{k T} D_{l}, \mu_{h}=\frac{e}{k T} D_{h}
$$

which follow from statistical mechanics. Here $e$ is the ionic charge and not the electronic charge. For a singly charged ion, $e$ is minus the charge on the electron. At room temperatures for singly charged ions the above formulas give

$$
D=.026 \mu\left(D \text { in } \frac{\mathrm{cm}^{2}}{\mathrm{sec}} ; \mu \text { in } \frac{\mathrm{cm}^{2}}{\mathrm{v}-\mathrm{sec}}\right)
$$

For a countercurrent adjusted to balance the slower ion one has, assuming

$$
\begin{gathered}
\mu_{l}-\mu_{h}=\frac{6.6 \times 10^{-4}}{200}:=3.3 \times 10^{-6} \frac{\mathrm{cm}^{2}}{\mathrm{v}-\mathrm{sec}} \\
a=3.3 \times 10^{-6} \mathrm{E}
\end{gathered}
$$

where $E$ is in $\mathrm{v} / \mathrm{cm}$. Hence

$$
\xi=\frac{3.3 \times 10^{-6}}{1.7 \times 10^{-5}} E x=0.2 E x .
$$

A change in $\xi$ by 1 corresponds in this case to a drop of $5 \mathrm{v}$ along the tube. More generally, for the same countercurrent adjustment, and singly charged ions

$$
\xi_{l}=\frac{\Delta \mu}{\mu} \frac{e E x}{k T}=39 \frac{\Delta \mu}{\mu} \text { times voltage drop }
$$

Here $\Delta \mu$ is the absolute value of the difference in mobilities. Thus if $\Delta \mu / \mu=1 / 1,000$ then $26 \mathrm{v}$ are required to change $\xi$ by unity and to give a factor, $e$, in the concentration in equilibrium.

The quantity $\tau$ can be considered as the time in units $D / a^{2}$. For $K^{+}$this unit is of the order

$$
\frac{1.7 \times 10^{-5}}{(3.3)^{2} \times 10^{-12} E^{2}}=1.6 \times 10^{6} \mathrm{sec} / E^{2}=430 \mathrm{hr} / E^{2}
$$

where $E$ is in $\mathrm{v} / \mathrm{cm}$ and the countercurrent adjustment is such as to keep one of the ions stationary. In the example of figure 5 one may consider $\tau \sim 2$ as corresponding to the time which it takes to reach half equilibrium, and the time constant is in this 
case of the order $1,000 \mathrm{hr} / E^{2}$. More generally the natural unit of time is

$$
\frac{D}{(\Delta \mu)^{2} E^{2}}
$$

On account of the large uncertainty in $\Delta \mu$ for uranium this unit cannot be computed with certainty. Since $\Delta \mu$ enters to the square, a decrease in $\Delta \mu$ by a factor 10 brings about an embarrassing increase of a factor 100 in the time unit. For a small cathode volume i. e. for small $\gamma$, the "natural" unit of time is the main thing that matters so that a sufficiently large $\Delta \mu$ is then especially important for a small time constant.

On the other hand, if $\gamma$ is large the value of $\tau$ corresponding to half equilibrium is much greater than unity as is seen in the examples following eq 9e. According to eq $8 \mathrm{c}$ for a fixed $\lambda$, the difference of mobility enters only once in the denominator in this case. For $K^{+}$assuming $\Delta \mu=\mu /{ }_{200}$ and taking $V=20 \mathrm{~cm}^{3}, A=1 \mathrm{~cm}^{2}$ the time constant is

$$
\frac{6 \times 10^{6} \text { sec. }}{E}\left(e^{\lambda}-1\right)=\frac{2,000 \mathrm{hr}}{E}\left(e^{\lambda}-1\right)
$$

It is interesting to note that if the equilibrium enrichment factor $e^{\lambda}$ is kept fixed and if $\gamma$ is large enough to make formula $8 \mathrm{~b}$ applicable then the time constant does not depend on the diffusion constant, $D$, but only on the difference of mobility $\Delta \mu$. In order, however, that $\lambda$ be fixed for different $D$ and $E$, one has to vary the length, $L$. For a tube $30 \mathrm{~cm}$ long with $90 \mathrm{v}$ applied having a free cross-sectional area $1 \mathrm{~cm}^{2}$ and a cathode volume $V=20 \mathrm{~cm}^{3}$, one has in the absence of convection and for $\Delta \mu / \mu=1 / 200, \lambda=17.5, \gamma=12$. An enormous equilibrium concentration of $e^{17}$ could be obtained in theory in such a case, but it would also take an impractical time to approach this equilibrium. It is doubtful, however, that $D$ can be as small as assumed $\left(1.7 \times 10^{-5} \frac{\mathrm{cm}^{2}}{\mathrm{sec}}\right)$ because of the convection currents.

The values $\lambda=2, \gamma=2.6995$, (used in example of $9 \mathrm{f}$ ) in the absence of convection for $\Delta \mu / \mu=1 / 200$ correspond to $10 \mathrm{v}$ applied to the tube. If the tube is $30 \mathrm{~cm}$ long and has a free cross-sectional area of $1 \mathrm{~cm}^{2}$, the cathode compartment would have to be $40 \mathrm{~cm}^{3}$. One has $a=3.3 \times 10^{-6} / 3=1.1 \times 10^{-6} \mathrm{~cm} /$ sec and $V / A=40 \mathrm{~cm}$ so that $T=3 \times 10^{8} \mathrm{sec} \sim 10^{5} \mathrm{hr}$ which is of the order of 5,000 days. If this tube were shortened to $3 \mathrm{~cm}$ keeping $V$ and $A$ constant and the same voltage then $\lambda$ remains unchanged and $a$ is increased by a factor 10 so that $T$ decreases to about 500 days.

There is a large gain in the shortened time constant due to shortening the tube for the same voltage. This is because of the increased current. The gain is of no real help except through the increased current and the detection of a difference in ionic mobilities appears to be easiest with a large current in a long tube.

\section{Equilibrium Concentration}

In this section the coefficients of diffusion will be decomposed into a part present in the stagnant liquid and a part due to spurious causes such as convection and stirring.

$$
D_{l}=D_{l}^{\circ}+\text { 丑, } D_{h}=D_{h}^{\circ}+\text { 目. }
$$

It is supposed that the spurious effects give the same contribution 且 to $D_{l}$ and $D_{h}$. The relations

$$
\mu_{l}=\frac{e}{k T} D_{l}^{\circ}, \mu_{h}=\frac{e}{k T} D_{h}^{\circ}
$$

hold. In equilibrium

where

$$
\frac{n_{l}}{n_{h}} \frac{n_{h}^{\circ}}{n_{l}^{\circ}}=e^{\alpha},
$$

$$
\begin{aligned}
& \frac{\alpha}{L}=\frac{\mu_{l} E+v}{\mathbf{⿴}+D_{l}^{\circ}}-\frac{\mu_{h} E+v}{\mathbf{3}+D_{h}^{\circ}}= \\
& \frac{D_{h}^{\circ}-D_{l}^{\circ}}{\left(\mathbf{⿴}+D_{l}^{\circ}\right)\left(\boldsymbol{⿴ 囗 十}+D_{h}^{\circ}\right)}\left(v-\frac{e E}{k T}\right. \text { 且) }
\end{aligned}
$$

The counter current is supposed to be adjusted so that

$$
v=-\mu_{h} E(1+\theta)
$$

If $\theta=0$, then isotope $h$ is kept stationary by the combined action of the countercurrent and of the migration velocity. However, $\theta$ will not be supposed to be necessarily zero. One has* from the above equations,

$$
\alpha=\frac{e \tilde{\mathbb{E}} L}{k T}\left[\mathbf{⿴}+D_{h}^{\circ}(1+\theta)\right] \frac{D_{l}^{\circ}-D_{h}^{\circ}}{\left(\mathbf{A}+D_{h}^{\circ}\right)\left(\mathbf{I}+D_{\imath}^{\circ}\right)}
$$

*Prof. H. C. Urey first derived a special case of the above formula which corresponds to $\mathbf{E}=0, \theta=0$. The authors are indebted to Prof. Urey for a stimulating discussion in this connection. 
For $\mathbb{\text { 刃 }}=0$ one has

$$
\begin{aligned}
\alpha= & \frac{e E L}{k T}(1+\theta) \frac{D_{l}^{\circ}-D_{h}^{\circ}}{D_{l}^{\circ}}= \\
& \frac{e E L}{k T}(1+\theta) \frac{\mu_{l}-\mu_{h}}{\mu_{l}}(\mathbb{B}=0)
\end{aligned}
$$

Here at room temperatures

$$
\frac{e E L}{k T}=39 x \text { applied voltage } \times \text { valence }
$$

just as for the quantity $\xi$. It is seen from the above formula for $\alpha$ that a large $\theta$ is advantageous for getting a large enrichment ratio. This fact should perhaps be emphasized because it indicates that it is better to have the countercurrent too strong rather than too weak if it is desired to test for the existence of a mobility effect.

If $\mathbb{\text { I }}>>D^{\circ}$, one has

$$
\alpha=\frac{e \tilde{E} L}{k T} \frac{D_{l}^{\circ}-D_{h}^{\circ}}{\text { 且 }}=\frac{e \tilde{E} L}{k T} \frac{\mu_{l}-\mu_{h}}{\mu_{l}} \frac{D^{\circ}}{\text { 且 }}\left(\text { 且 }>>D^{\circ}\right)
$$

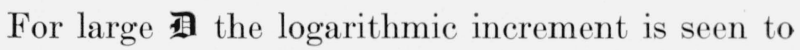
be a fraction $D^{\circ}$ 国 of its value for $\mathbf{\theta}=0, \theta=0$. In this limit the exact adjustment of the counter current is of no importance for the enrichment factor at equilibrium.

\section{References}

[1] A. Keith Brewer, S. L. Madorsky, and J. W. Westhaver, Science 104, 156 (1946).

[2] A. Keith Brewer, S. L. Madorsky, John Keenan Taylor, Vernon H. Dibeler, J. Research NBS 38, 137 (1947) RP1765.

[3] Samuel L. Madorsky and Sidney Straus, J. Research NBS 38, 169 (1947) RP1766.

[4] Jahncke, Emde, Tables of functions with formulae and curves, p. 3 (B. G. Teubner, Leipzig, 1909).

Washington, October 25, 1946 